\title{
The Impact of a Software Tool that Supports a Part-Complete Solution Method on the Learning Outcomes of Novice Programmers
}

\author{
Stuart Garner \\ Edith Cowan University, Joondalup, Australia
}

\section{s.garner@ecu.edu.au}

\begin{abstract}
This paper reports on the findings from a quantitative research study into the use of a software tool that was built to support a part-complete solution method (PCSM) for the learning of computer programming. The use of part-complete solutions to programming problems is one of the methods that can be used to reduce the cognitive load that students experience during their learning.

The tool that was built is a code restructuring tool known as CORT. An inquiry into its effect on student learning outcomes took place over a period of 14 weeks at an Australian university.

Two student groups participated in an introductory programming unit, one of which utilized the CORT system whilst the other acted as a control group. No significant difference were found in the relative achievement of students in the CORT and non-CORT groups in tests of programming achievement. However the results showed significant advantages for the CORT students in terms of time saving and levels of tutor help required. Differences also emerged in levels of achievement between certain sub-groups, these being age, computer literacy and previous programming experience.
\end{abstract}

Keywords: learning, programming, cognition. 